\title{
Does Emotional Labor Increase the Risk of Suicidal Ideation among Firefighters?
}

\author{
Dae-Sung Hyun ${ }^{1,2}$, Da-Yee Jeung ${ }^{3}$, Changsoo Kim ${ }^{4}$, Hye-Yoon Ryu ${ }^{2,5}$, and Sei-Jin Chang ${ }^{2,5}$ \\ ${ }^{1}$ Departments of Biostatistics and Computing, Graduate School, Yonsei University, Seoul; \\ ${ }^{2}$ Department of Preventive Medicine, Yonsei University Wonju College of Medicine, Wonju; \\ ${ }^{3}$ Department of Dental Hygiene, Hanyang Women's University, Seoul; \\ ${ }^{4}$ Department of Preventive Medicine, Yonsei University College of Medicine, Seoul; \\ ${ }^{5}$ Institute of Occupational and Environment Medicine, Yonsei University Wonju College of Medicine, Wonju, Korea.
}

Purpose: To investigate whether emotional labor is associated with suicidal ideation in Korean firefighters.

Materials and Methods: Data were obtained from the Firefighter Research: Enhancement of Safety \& Health (FRESH) Study, which was designed to investigate the effects of job characteristics on mental and physical health among Korean firefighters. A total of 18101 firefighters were chosen from a nationwide sample. The Korean Emotional Labor Scale (K-ELS) was used to evaluate exposure to emotional labor, which consisted of five sub-factors: emotional demand and regulation, overload and conflict in customer service, emotional disharmony and hurt, organizational surveillance and monitoring, and lack of a supportive and protective system in the organization.

Results: Firefighters who were in the risk group were more likely to experience suicidal ideation than those in the normal group for each of the five sub-scales of emotional labor. The estimated mean values for suicidal ideation in the risk group were significantly higher than those in the normal group: 1.667 (95\% CI: 1.344-2.069) for emotional demand and regulation, 1.590 (95\% CI: 1.2432.033) for overload and conflict in customer service, 2.409 (95\% CI: 1.954-2.969) for emotional disharmony and hurt, 2.214 (95\% CI: 1.832-2.676) for organizational surveillance and monitoring, and 1.665 (95\% CI: 1.387-1.999) for lack of a supportive and protective system in the organization.

Conclusion: These results suggest that experience and exposure to chronic and excessive emotional labor might play a crucial role in the development of suicidal ideation among firefighters.

Key Words: Emotional labor, suicidal ideation, firefighter

\section{INTRODUCTION}

When interacting with customers, service workers may be required to manage the expression of their own feelings in carrying out their job. These emotional interactions with customers

Received: October 8, 2019 Revised: December 5, 2019

Accepted: December 26, 2019

Corresponding author: Sei-Jin Chang, PhD, Department of Preventive Medicine, Yonsei University Wonju College of Medicine, 20 Ilsan-ro, Wonju 26426, Korea. Tel: 82-33-741-0343, Fax: 82-33-747-0409, Email: chang0343@yonsei.ac.kr

-The authors have no potential conflicts of interest to disclose.

(C) Copyright: Yonsei University College of Medicine 2020

This is an Open Access article distributed under the terms of the Creative Commons Attribution Non-Commercial License (https://creativecommons.org/licenses/ by-nc/4.0) which permits unrestricted non-commercial use, distribution, and reproduction in any medium, provided the original work is properly cited. may occur directly or indirectly. Emotional labor occurs when emotion work is required in exchange for something, such as wages or some other type of valued compensation. Wharton ${ }^{1}$ proposed that these actions are not only performed for a wage, but they may also be under the control of others.

Emotional laborers are more likely to hide their real emotions because their emotional expressions while performing workrelated duties should be displayed according to organizational goals and guidelines. However, these chronic interactions involving restricted emotional expression with customers may lead to both physical and mental depletion. Indeed, emotional labor has been shown to be associated with physical and mental health problems ${ }^{2}$ due to surface acting (the act of suppressing or hiding one's own feelings that occur during work to express positive emotions) and deep acting (where individuals try 
to consciously change their feelings to truly express positive emotions, rather than give a superficial appearance of changed feelings). ${ }^{3}$ Mental health problems resulting from emotional labor among service workers have been widely reported. In Korea, recent studies have documented that emotional laborers, such as nurses, dental hygienists, call center workers, sales workers, bankers, and hoteliers, are at high risk for depressive symptoms and burnout. ${ }^{4,5}$ Emotional expression appearing as surface acting in reflection of organizational goals, rather than individual spontaneity, can affect depressive symptoms and burnouts, ${ }^{6,7}$ and depressive symptoms and burnout have been found to be strongly associated with suicide attempts. ${ }^{8,9}$ Further, about $60 \%$ of suicide deaths have been shown to be associated with major depressive disorder or a mood disorder. ${ }^{10}$

Suicide-related deaths are steadily increasing. Currently, more than 800000 people worldwide die each year from suicide. ${ }^{11}$ Suicide is reported to be the second cause of death among workers aged 15 to 29 years. ${ }^{12}$ From 2012 to 2016 , the occupations with the highest number of suicides were service and sales associates. ${ }^{13}$ This is especially concerning as the service industry continues to expand and strengthen, making the risk of suicide for service workers an important issue.

As the duties of firefighters have expanded from fire prevention and suppression to related tasks, such as rescue and emergency service, the proportion of interpersonal tasks has been increasing. In addition, firefighters may face civilians who are exposed to feelings of loss, exposure of death, mental trauma, and anger in fire, rescue, and emergency situations. Even if firefighters are trained in the appropriate procedures for each situation, they may not be emotionally prepared for nervous or stressful situations or interactions involving customer service. In this way, firefighters experiencing confrontations with civilians are exposed to emotional labor. According to the occupational status survey of emotional labor, the emotional labor score of fire officials was 3.74 out of 5 points, and they were classified as a high risk occupational group for emotional labor. ${ }^{14}$ Firefighters were more likely to experience emotional labor than other occupational groups (e.g., manufacturing workers).

Although there has been a significant change in the psychological health of firefighters, there are few studies on the relationship between emotional labor and suicide in firefighters. Therefore, the present study aimed to examine the relationship between emotional labor and suicidal ideation for firefighters.

\section{MATERIALS AND METHODS}

\section{Study participants}

The participants in this study included individuals who completed the online survey "Job Contents And Health Survey of Firefighters" conducted for the FRESH (Firefighter Research: Enhancement of Safety \& Health) Study entitled "The Study on Physical and Mental Health Promotion of Firefighters through
Lifetime Health Risk Management System in Korean Firefighters." Of 19203 total respondents who participated, 18101 firefighters were included for the final analysis of this study. We excluded 1102 individuals who did not have complete responses (Fig. 1). This study was approved by the Institutional Review Board of Yonsei University Wonju College of Medicine (Approval No. CR316014).

\section{Measures}

The data for this study were collected using an online-based, self-report questionnaire. All participants were asked about general characteristics, occupational characteristics, characteristics of past work, job stress, emotional labor, working violence, health behavior, and health status.

\section{Emotional labor}

Emotional labor was measured using the Korean Emotional Labor Scale (K-ELS), which was designed to evaluate the work environment and factors related with emotional labor. The emotional labor questionnaires consist of 24 questions in five areas including, 1) emotional demands and regulation (5 items), 2) overload and conflict of customer service (3 items), 3) emotional disharmony and hurt (6 items), 4) organizational surveillance and monitoring (3 items), and 5) lack of a supportive and protective system in the organization (7 items). Each item on emotional labor was rated on a 4-point Likert scale (1=disagree completely, 2=disagree, $3=$ =agree, $4=$ =agree completely). The five subfactors were converted into a score from $0-100$. Higher scores represented a higher intensity of emotional labor. Results of reliability analysis for the five sub-factors in the original study showed Cronbach's $\alpha$ values ranging from 0.761 to $0.904 .{ }^{15}$ Cronbach's $\alpha$ for the five sub-factors in this study ranged from 0.772 to 0.928 . In this study, the five sub-factors were further divided into a normal and risk group by using cut points determined according to gender.

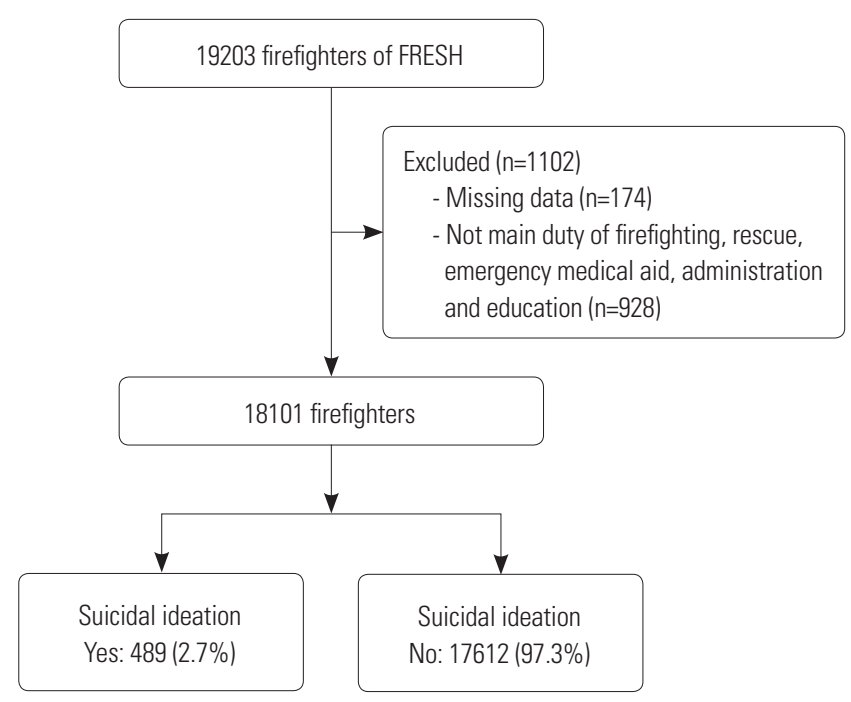

Fig. 1. Recruitment process of the study subjects. 


\section{Suicidal ideation}

Suicidal ideation was measured by asking, "(In the past 12 months) Have you thought about committing suicide?" There was a dichotomous response category (yes vs. no).

\section{Statistical analysis}

To compare suicidal ideation according to general characteristics, occupational characteristics, health behavior, health status, trauma exposure, and emotional labor, we conducted independent two-sample t-tests and chi-square tests. A multivariate Poisson regression model was used to evaluate the independent association of emotional labor with suicidal ideation. All analyses adjusted for several covariates, such as age, gender, education, marital status, main duty, shift work, smoking, drinking alcohol, participant health status, and trauma exposure. A few firefighters reported that they have thoughts of "committing suicide." When facing such rare events in data analysis, Poisson analysis is very useful. ${ }^{16}$ The results are expressed as relative risks (RRs) with 95\% confidence intervals (CI). $p<0.05$ was considered statistically significant, and all statistical analysis were performed using SPSS (version 23.0; IBM Corp., Armonk, NY, USA).

\section{RESULTS}

\section{General characteristics of the participants}

Among the participants in this study, 17028 (94.1\%) were male, $8148(45.0 \%)$ graduated from a 4-year college, and $13170(72.8 \%)$ were married. The main duties of the participants were firefighting (49.1\%), emergency medical aid (28.4\%), administration or education (11.5\%), and rescue (10.9\%). The most frequent shift work was three shifts ( $\mathrm{n}=14213,78.5 \%)$. Of the total study sample, 5744 (31.7\%) were current smokers, 2470 (13.6\%) were drinking three times a week, and 2152 (11.9\%) reported that their subjective health status was unhealthy.

\section{Intensity of emotional labor in firefighters}

The distributions of participants in the risk group for each of the five sub-factors of emotional work were $11.7 \%$ for emotional demands and regulation, $8.5 \%$ for overload and conflict of customer service, $9.7 \%$ for emotional disharmony and hurt, $17.9 \%$ for organizational surveillance and monitoring, and $36.2 \%$ for lack of a supportive and protective system in the organization.

\section{Differences in suicidal ideation according to general characteristics of the study participants}

Of the 18101 firefighters, 489 (2.7\%) reported that they have thoughts of committing suicide. Suicidal ideation was more common among firefighters who were divorced, bereaved, and separated (5.1\%), compared to those who were unmarried (2.1\%) and married $(2.9 \%)(p<0.001)$. The proportion of participants with suicidal ideation by main duty was higher for administrative or education (3.3\%) than for firefighting (2.9\%), rescue
(2.4\%), and emergency medical aid $(2.2 \%)(p=0.030)$. Participants who were smoking (3.3\%) and drinking more than three times a week (5.1\%) showed a higher proportion of suicidal ideation than their counterparts $(p<0.001)$. For the five sub-factors of emotional labor, all of the risk groups had significantly more suicidal ideation than the normal groups: emotional demands and regulation, $5.4 \%(p<0.001)$; overload and conflict in customer service, $5.3 \%$ ( $p<0.001)$; emotional disharmony and hurt, $7.7 \%(p<0.001)$; organizational surveillance and monitoring, $5.8 \%(p<0.001)$; and lack of a supportive and protective system in the organization, $4.2 \%(p<0.001)$ (Table 1$)$.

\section{Relationship between emotional labor and suicidal ideation}

A multivariate Poisson regression analysis was performed to examine the relationship between the five sub-factors of emotional labor and suicidal ideation after adjusting for age, gender, education level, marital status, main duty, shift work, smoking, drinking alcohol, subjective health status, and trauma exposure. The relative risk values for suicidal ideation in the risk group were significantly higher than those of the normal group: 1.667 (95\% CI: 1.344-2.069) for emotional demands and regulation, 1.590 (95\% CI: 1.243-2.033) for overload and conflict in customer service, 2.409 (95\% CI: 1.954-2.969) for emotional disharmony and hurt, 2.214 (95\% CI: 1.832-2.676) for organizational surveillance and monitoring, and 1.665 (95\% CI: 1.3871.999) for lack of a supportive and protective system in the organization (Table 2).

\section{DISCUSSION}

The present study aimed to examine the relationships between emotional labor and suicidal ideation for firefighters. Our findings indicated that participants in the risk groups for each of the five sub-factors of emotional work comprised $11.7 \%$ for emotional demands and regulation, $8.5 \%$ for overload and conflict of customer service, $9.7 \%$ for emotional disharmony and hurt, $17.9 \%$ for organizational surveillance and monitoring, and $36.2 \%$ for lack of a supportive and protective system in the organization. Previous studies have documented that a lower degree of organizational commitment ${ }^{17}$ and higher frequency of emotional expression and emotional dissonance are associated with lower job satisfaction..$^{18}$ Among firefighters at the Seoul Emergency Operations Center, social support had a mediating effect on the relationship between emotional labor and job performance. ${ }^{19}$ The emotional labor of firefighters has also been found to have a major risk factor affecting mental health ${ }^{20}$ and to have a negative effect on turnover intentions. ${ }^{21}$ In addition, emotional labor was a major predictor of reduced work efficiency and increased work disability for firefighters. Because firefighters must meet various demands from civilians, job stress and posttraumatic stress can result in severe adverse consequences, such as de- 
Table 1. Characteristics of the Study Population by Presence of Suicidal Ideation ( $n=18101$ )

\begin{tabular}{|c|c|c|c|}
\hline \multirow{2}{*}{ Variables } & \multicolumn{2}{|c|}{ Suicidal ideation } & \multirow{2}{*}{$p$ value } \\
\hline & Yes $(n=489,2.7 \%)$ & No $(n=17612,97.3 \%)$ & \\
\hline Gender & & & 0.041 \\
\hline Male & $449(2.6)$ & $16579(97.4)$ & \\
\hline Female & $40(3.7)$ & $1033(96.3)$ & \\
\hline Education & & & 0.268 \\
\hline Less than or equal to high school graduate & $132(2.9)$ & $4371(97.1)$ & \\
\hline Junior college graduate & $132(2.4)$ & $5318(97.6)$ & \\
\hline 4-year college graduate or more & $225(2.8)$ & $7923(97.2)$ & \\
\hline Marital status & & & 0.001 \\
\hline Unmarried & $97(2.1)$ & $4597(97.9)$ & \\
\hline Married & $380(2.9)$ & $12790(97.1)$ & \\
\hline Divorce/bereavement/separation & $12(5.1)$ & $225(94.9)$ & \\
\hline Main duty & & & 0.030 \\
\hline Firefighting & $259(2.9)$ & $8634(97.1)$ & \\
\hline Rescue & $47(2.4)$ & $1924(97.6)$ & \\
\hline Emergency medical aid & $115(2.2)$ & $5033(97.8)$ & \\
\hline Administration or education & $68(3.3)$ & $2021(96.7)$ & \\
\hline Shift work & & & 0.222 \\
\hline No shift & $69(3.1)$ & $2138(96.9)$ & \\
\hline 24-hour shift & $15(2.7)$ & $535(97.3)$ & \\
\hline Two shifts & $9(2.6)$ & $333(97.4)$ & \\
\hline Three shifts & $384(2.7)$ & $13829(97.3)$ & \\
\hline Other & $12(1.5)$ & 777 (98.5) & \\
\hline Smoking & & & $<0.001$ \\
\hline Yes & $192(3.3)$ & $5552(96.7)$ & \\
\hline No & $297(2.4)$ & $12060(97.6)$ & \\
\hline Alcohol drinking & & & $<0.001$ \\
\hline No & $137(2.6)$ & $5064(97.4)$ & \\
\hline One or two times a week & $225(2.2)$ & $10205(97.8)$ & \\
\hline More than three times a week & $127(5.1)$ & $2343(94.9)$ & \\
\hline Subjective health status & & & $<0.001$ \\
\hline Healthy & $75(0.9)$ & $8119(99.1)$ & \\
\hline Moderate & $208(2.7)$ & 7547 (97.3) & \\
\hline Unhealthy & $206(9.6)$ & $1946(90.4)$ & \\
\hline \multicolumn{4}{|l|}{ Korean Emotional Labor Scale (K-ELS) } \\
\hline Emotional demands and regulation & & & $<0.001$ \\
\hline Normal & $375(2.3)$ & $15607(97.7)$ & \\
\hline Risk & $114(5.4)$ & $2005(94.6)$ & \\
\hline Overload and conflict in customer service & & & $<0.001$ \\
\hline Normal & $407(2.5)$ & $16154(97.6)$ & \\
\hline Risk & $82(5.3)$ & $1458(94.7)$ & \\
\hline Emotional disharmony and hurt & & & $<0.001$ \\
\hline Normal & $354(2.2)$ & $15998(97.8)$ & \\
\hline Risk & $135(7.7)$ & $1614(92.3)$ & \\
\hline Organizational surveillance and monitoring & & & $<0.001$ \\
\hline Normal & $300(2.0)$ & $14565(98.0)$ & \\
\hline Risk & $189(5.8)$ & $3047(94.2)$ & \\
\hline Lack of a supportive and protective system in the organization & & & $<0.001$ \\
\hline Normal & $215(1.9)$ & $11332(98.1)$ & \\
\hline Risk & $274(4.2)$ & $6280(95.8)$ & \\
\hline Age (yr) & $42.52 \pm 9.09$ & $40.92 \pm 9.14$ & $<0.001$ \\
\hline
\end{tabular}

Data are presented as number $(\%)$ or mean \pm SD. 
Table 2. Results of Poisson Regression Analyses for Suicidal Ideation

\begin{tabular}{lccc}
\hline & Crude & Model 1 & \multicolumn{1}{c}{ Model 2 $^{\dagger}$} \\
\cline { 2 - 4 } & RR (95\% Cl) & RR (95\% CI) & RR (95\% CI) \\
\hline Emotional demands and regulation & $2.293(1.859-2.828)$ & $2.340(1.892-2.894)$ & $1.667(1.344-2.069)$ \\
Overload and conflict in customer service & $2.167(1.709-2.747)$ & $2.269(1.778-2.894)$ & $1.590(1.243-2.033)$ \\
Emotional disharmony and hurt & $3.565(2.924-4.347)$ & $3.686(3.006-4.521)$ & $2.409(1.954-2.969)$ \\
Organizational surveillance and monitoring & $2.894(2.412-3.472)$ & $2.947(2.448-3.547)$ & $2.214(1.832-2.676)$ \\
Lack of a supportive and protective system in the organization & $2.245(1.878-2.684)$ & $2.178(1.819-2.607)$ & $1.665(1.387-1.999)$ \\
\hline
\end{tabular}

$\mathrm{RR}$, relative risk; $\mathrm{Cl}$, confidence interval.

*Model 1: adjustment for age, gender, education, marital status, ${ }^{\dagger}$ Model 2: adjustment for Model 1+shift work, smoking, drinking alcohol, subjective health status.

pression, burnout, and suicide. ${ }^{22-24}$ In 2015,12 Korean firefighters died due to apparent suicide, which is six times higher than the number of firefighters who died due to accidents while working. The number of firefighters who committed suicide during the past 5 years was 41 , which was higher than the number of firefighters who died while performing their duties.

Multivariate Poisson analysis showed that the five sub-scales of emotional labor affected suicidal ideation in this study. The RRs for suicidal ideation were more than approximately two times higher in the risk group than in the normal group for the sub-scales of overload and conflict in customer service and organizational surveillance and monitoring. Emotional disharmony and hurt are the items that measured the emotional disharmony caused by the clash between the actual emotions of the worker and the emotional expression norms required by the workplace, and the degree of damage to the various types of minds during customer interactions. Emotional dissonance is well known to have a negative impact on emotional workers. ${ }^{25}$

The organizational culture of firefighters reflects an army-like class structure, and they have a strict sub-culture, compared to other occupational groups. The higher proportions of risk groups associated with the emotional demands on firefighters may be due to the hierarchical organizational climate of the firefighters. Among the sub-factors of emotional labor, organizational surveillance and monitoring, a sub-factor related to the organization, was a measure of the degree to which workers felt that it was hard to monitor how well they responded to customers and applied it unilaterally to performance appraisal and evaluation. ${ }^{15}$ An expansion in the monitoring system has resulted in workers with increasing emotional stress in the workplace ${ }^{26}$ and increased fatigue during their working hours. ${ }^{27}$ In addition, lack of a supportive and protective system in the organization is a measure of the workplace support system that can be used to alleviate problems and can facilitate progress for organizational management plans or actions when a problem occurs for the customer. ${ }^{15}$ Studies have shown a positive effect on emotional labor when the supportive culture of an organization is high. ${ }^{28}$ In particular, officials of government organizations tend to underestimate emotional labor. ${ }^{29}$ In Korea, it has been reported that emotional expression of officials is suppressed and that evaluation thereof is unnecessary. ${ }^{30}$

Possible mechanisms for the link between emotional labor and suicidal ideation have been documented. Suppressing emotions while performing emotional service can result in the loss of personal resources. ${ }^{31}$ According to the Conservation of Resources theory, ${ }^{32}$ the loss or threat of personal resources increases physiological arousal and health problems. Suppressing emotions requires more energy resources, as exhibited by increased physiological arousal and higher levels of glucose..$^{33}$ As a result, persistent exposure to stress due to excessive and chronic emotional demands might activate the stress system, including the hypothalamic pituitary adrenal axis and the sympathetic nervous system. Furthermore, if the demand for emotion regulation is excessive and persistent, it can lead to depression. ${ }^{34}$

Another mechanisms can be discussed in that surface acting results in emotional burnout due to efforts to fake or suppress negative emotions, ${ }^{35}$ which can contribute to health deterioration. ${ }^{36}$ Surface acting can lead to energy deficits from persistent internal tension due to emotional dissonance resulting from a collision between the behavior of suppressing one's feelings and the emotions. The continuing experience of emotional dissonance can play a crucial impact on the loss or threat of resources and eventually to exhaustion. ${ }^{37}$

Depression and burnout caused by emotional labor are closely related to suicide and suicide attempts and are known to be important risk factors. ${ }^{38}$ Of the total study participants, 489 (2.7\%) answered that they considered attempting suicide in the past year.

This study has several strengths. First, the relationship between individual sub-factors and suicidal ideation was examined using the Korean Emotional Labor Scale standardized with consideration for the socio-cultural context and the qualitative aspect of emotion in Korea. Second, the relationship between emotional labor and suicidal ideation in firefighters, which is a recent issue, was confirmed through a Poisson regression analysis. Third, this study was analyzed using a nationwide survey that was completed by $50 \%$ of all firefighters in Korea. Fourth, findings from this study can be used as a basis for the necessity of an intervention program with which to examine and reduce suicidal ideation in firefighters to prevent suicidal behavior.

There are some potential limitations to this study. First, survey completion methods may have varied because it was completed online. Second, because the design of this study was crosssectional, no causal relationship between emotional labor and 
suicidal ideation can be specified. Further, it is necessary to conduct prospective studies to confirm the association between emotional labor and suicide. Additionally, it is not clear whether suicidal ideations were caused by emotional labor during work because questionnaire items of suicidal ideation concerned the past 12 months. However, a recent study showed that long lasting exposure to emotional labor in the short term cannot be ignored, although in general, negative emotions, such as emotional dissonance, can be generated by accumulated emotional labor. ${ }^{39}$

In conclusion, our findings suggest that emotional labor is associated with suicidal ideation in Korean firefighters. Emotional labor is not limited to the service industry and extends to all sectors of industry. Therefore, additional research is required to examine emotional workers from several service industries, including face-to-face service businesses. This study confirmed the relationship between emotional labor and suicidal ideation found in previous studies on firefighters who are emotional workers in the public service sector. This result suggests that continuous exposure and experience of emotional labor in the work of a firefighters play a role in increasing suicidal ideation. The results of this study can contribute to suicide prevention for firefighters by informing mental health promotion management programs to reduce the negative influence of emotional labor on firefighters.

\section{ACKNOWLEDGEMENTS}

This research was supported by the Fire Fighting Safety \& 119 Rescue Technology Research and Development Program funded by National Fire Agency (MPSS-2015-80).

\section{AUTHOR CONTRIBUTIONS}

Conceptualization: Dae-Sung Hyun and Sei-Jin Chang. Data curation: Dae-Sung Hyun. Formal analysis: Dae-Sung Hyun, Da-Yee Jeung, and Hye-Yoon Ryu. Funding acquisition: Changsoo Kim and Sei-Jin Chang. Investigation: Dae-Sung Hyun, Da-Yee Jeung, and SeiJin Chang. Methodology: Dae-Sung Hyun and Sei-Jin Chang. Project administration: Sei-Jin Chang and Hye-Yoon Ryu. Resources: DaeSung Hyun and Da-Yee Jeung. Software: Dae-Sung Hyun and Hye Yoon Ryu. Supervision: Sei-Jin Chang and Changsoo Kim. Validation: Sei-Jin Chang. Visualization: Dae-Sung Hyun. Writing_original draft: Dae-Sung Hyun and Sei-Jin Chang. Writing_review \& editing: Sei-Jin Chang. Approval of final manuscript: all authors.

\section{ORCID iDs}

Dae-Sung Hyun Da-Yee Jeung Changsoo Kim Hye-Yoon Ryu Sei-Jin Chang https://orcid.org/0000-0002-3911-3913 https://orcid.org/0000-0003-4730-8814 https://orcid.org/0000-0002-5940-5649 https://orcid.org/0000-0002-6695-8313 https://orcid.org/0000-0001-9347-3592

\section{REFERENCES}

1. Wharton AS. The affective consequences of service work: managing emotions on the job. Work Occup 1993;20:205-32.

2. Kim HJ, Choo J. Emotional labor: links to depression and work-related musculoskeletal disorders in call center workers. Workplace Health Saf 2017;65:346-54.

3. Hochschild AR. The managed heart: commercialization of human feeling. Berkeley (CA): University of California Press; 2012.

4. Kim GH, Lee HS, Jung SW, Lee JG, Lee JH, Lee KJ, et al. Emotional labor, workplace violence, and depressive symptoms in female bank employees: a questionnaire survey using the K-ELS and K-WVS. Ann Occup Environ Med 2018;30:17.

5. Jeung DY, Lee HO, Chung WG, Yoon JH, Koh SB, Back CY, et al. Association of emotional labor, self-efficacy, and type A personality with burnout in Korean dental hygienists. J Korean Med Sci 2017; 32:1423-30.

6. Grandey AA. When "the show must go on": surface acting and deep acting as determinants of emotional exhaustion and peer-rated service delivery. Acad Manage J 2003;46:86-96.

7. Kim SY, Chang SJ, Kim HR, Roh JH. A study on the relationship between emotional labor and depressive symptoms among Korean industrial service employees. Korean J Occup Environ Med 2002; 14:227-35

8. Galán F, Ríos-Santos JV, Polo J, Rios-Carrasco B, Bullón P. Burnout, depression and suicidal ideation in dental students. Med Oral Patol Oral Cir Bucal 2014;19:e206-11.

9. Dyrbye LN, Thomas MR, Massie FS, Power DV, Eacker A, Harper W, et al. Burnout and suicidal ideation among U.S. medical students. Ann Intern Med 2008;149:334-41.

10. Cavanagh JT, Carson AJ, Sharpe M, Lawrie SM. Psychological autopsy studies of suicide: a systematic review. Psychol Med 2003; 33:395-405

11. World Health Organization. Suicide data [accessed on 2019 June 15]. Available at: https://www.who.int/mental_health/prevention/suicide/suicideprevent/en/.

12. World Health Organization. Preventing suicide: a global imperative. Geneva: World Health Organization; 2014.

13. Ministry of Health and Welfare, Korea Suicide Prevention Center. 2018 Suicide prevention white book. Seoul: Korea Suicide Prevention Center; 2019.

14. Han SG, Park CS, Jeong YK, Chang HJ, Kim NR. The study of Korean Occupational Index (2012). Seoul: Korea Research Institute for Vocational Education \& Training; 2012.

15. Chang SJ, Kang HT, Kim SY, Kim IA, Kim JI, Kim HR, et al. Application study of Korean Emotional Labor Scale and Korean Workplace Violence Scale. Ulsan: Occupational Safety and Health Research Institute; 2014.

16. Warner P. Poisson regression. J Fam Plann Reprod Health Care 2015;4:223-4.

17. Joo KS. A study on emotional labor and organizational commitment among firefighters in Korea. Crisisonomy 2017;13:45-54.

18. Cho S, Ryu SI, Lee MK. A study on emotional labor and job satisfaction among firefighters in Korea. Crisisonomy 2017;13:153-60.

19. Park CS, Kim JK. The influence of emotional labor at Seoul Management Operations Center on job performance: focusing on the mediating effect of social support. J Korea Saf Manag Sci 2015;17: 13-25.

20. Kim YJ, Kim JH, Shim GS. The relationship among job stress, emotional labor, resilience and mental health in firefighters. J Korea Converg Soc 2017;8:379-89.

21. Ji DH, Choi MS. The comparative study on changes in turnover 
intention according to the level of burn out and the convergence mediating factors in fire officer. J Korea Soc Computer Inf 2016; 21:111-7.

22. Smith TD, Hughes K, DeJoy DM, Dyal MA. Assessment of relationships between work stress, work-family conflict, burnout and firefighter safety behavior outcomes. Saf Sci 2018;103:287-92.

23. Katsavouni F, Bebetsos E, Malliou P, Beneka A. The relationship between burnout, PTSD symptoms and injuries in firefighters. Occup Med (Lond) 2016;66:32-7.

24. Boffa JW, Stanley IH, Smith LJ, Mathes BM, Tran JK, Buser SJ, et al. Posttraumatic stress disorder symptoms and suicide risk in male firefighters: the mediating role of anxiety sensitivity. J Nerv Ment Dis 2018;206:179-86.

25. Ashforth BE, Humphrey RH. Emotional labor in service roles: the influence of identity. Acad Manage Rev 1993;18:88-115.

26. Kim WB, Rhee KY, Lee GR. Work environment and stress of emotional laborers. Korean J Sociol 2012;46:123-49.

27. Smith MJ, Carayon P, Sanders KJ, Lim SY, Legrande D. Employee stress and health complaints in jobs with and without electronic performance monitoring. Appl Ergon 1992;23:17-27.

28. Choi SW. Cultural orientation of emotional labor in administrative service organizations: the case of national tax officials. Korean Public Adm Rev 2012;46:79-101.

29. Meier KJ, Mastracci SH, Wilson K. Gender and emotional labor in public organizations: an empirical examination of the link to performance. Public Adm Rev 2006;66:899-909.

30. Kim SG. An exploratory study on the emotional labor in public sector: focused on street-level bureaucrats in the civil service system.
Korean J Local Gov Stud 2009;13:51-70.

31. Diefendorff JM, Richard EM, Yang J. Linking emotion regulation strategies to affective events and negative emotions at work. J Vocat Behav 2008;73:498-508.

32. Hobfoll SE, Freedy J. Conservation of resources: a general stress theory applied to burnout. In: Schaufeli WB, Maslach C, Marek T, editors. Professional burnout: recent developments in theory and research. Washington DC: Taylor \& Francis; 1993. p.115-29.

33. Baumeister RF, Vohs KD, Tice DM. The strength model of self-control. Curr Dir Psychol Sci 2007;16:351-5.

34. Yoon SL, Kim JH. Job-related stress, emotional labor, and depressive symptoms among Korean nurses. J Nurs Scholarsh 2013;45: 169-76.

35. Brotheridge CM, Grandey AA. Emotional labor and burnout: comparing two perspectives of "people work". J Vocat Behav 2002;60: 17-39.

36. Yagil $\mathrm{D}$. The mediating role of engagement and burnout in the relationship between employees' emotion regulation strategies and customer outcomes. Eur J Work Organ Psychol 2012;21:150-68.

37. Grandey AA, Fisk GM, Steiner DD. Must "service with a smile" be stressful? The moderating role of personal control for American and French employees. J Appl Psychol 2005;90:893-904.

38. Dunnette MD, Hough LM. Handbook of industrial and organizational psychology, Vol. 3. 2nd ed. Palo Alto (CA): Consulting Psychologists Press; 1992.

39. Kim MS, Kang JW, Han SS. Emotional labor and work life satisfaction of department store workers: mediating effect of job oppression. J Korean Soc Occup Environ Hyg 2019;29:420-9. 